\title{
A Nonspecific Primer Anchored PCR Technique for Chromosome Walking
}

\author{
Yan Zhang, Daqun Liu*, Wenxiang Yang, Yaning Li and Hongfei Yan \\ College of Plant Protection; Agricultural University of Hebei; Biological Control Center for Plant Diseases and \\ Plant Pests of Hebei Province; Baoding 071001; China
}

\begin{abstract}
A chromosome walking method was improved in this work. The new method was named as nonspecific primer anchored PCR (NPA-PCR). Nested gene specific primers were designed based on the known region and long random primer using degeneracy oligonucleotides for nonspecific anchoring. Annealing temperatures were varied to control the priming. Target sequences were obtained by PCR with random primer and gene-specific primer. Nonspecific sequence with long random primers at both ends formed stem loop structure due to inverted terminal repeats. The method was employed to isolate a gene with newly-isolated actinomycin producing strain Streptomyces setonii Z-L-22. A $0.77 \mathrm{~kb}$ fragment of actinomycin synthetase gene cluster was isolated from the strain. The fragments of $1474 \mathrm{bp}$ and $701 \mathrm{bp}$ were obtained, respectively, at the up and down streams of known fragment through the this method. NCBI Blast analysis showed that the walking sequence and the known sequence were located conjointly in the same cluster gene. It demonstrated that the result was correct and this technique could be useful and efficient for chromosome walking or isolating the gene.
\end{abstract}

Key words: nonspecific primer anchored PCR; chromosome walking; random primer

\section{INTRODUCTION}

The hunt for missing sequence data is often required in the experiments. Several polymerase chain reactions (PCR)-based methodologies are available for walking from a known region to cloned or uncloned genomic DNA, including inverse PCR (IPCR) (Triglia et al., 1988; Melanie et al.,2004, Ochman et al.,1988), adaptor ligation (LM-PCR) (Espelund and Jakobsen, 1992; Kilstrupand Kristiansen, 2000; Yan et al.,2003;

Chen et al., 2004) and randomly primed PCR (RPPCR). The first two methods have high specificity but require enzyme digestion and ligation, making them relatively expensive and time-consuming. Thermal asymmetric interlaced PCR (TAIL-PCR) (Liu et al., 1995) is a representative method of the third one. This method has its specific advantage but the amplified products are generally limited to a length of $<1 \mathrm{~kb}$.

Here we report a nonspecific primer anchored PCR (NPA-PCR), which has been developed on the basis of Peng's (Peng, 2005) nonspecific anchoring of suppression sequence PCR (NASSPCR).

\footnotetext{
*Author for correspondence: ldq@hebau.edu.cn
} 
Table 1 - Reactions of NPA-PCR and NASS-PCR.

\begin{tabular}{|c|c|c|c|c|c|c|}
\hline \multirow[b]{2}{*}{ steps } & \multicolumn{2}{|c|}{ NPA-PCR } & \multirow[b]{2}{*}{ steps } & \multicolumn{3}{|c|}{$\begin{array}{l}\text { NASS-PCR } \\
\end{array}$} \\
\hline & Primers adding & cycles procedure & & Primers adding & cycl & es procedure \\
\hline \multirow{4}{*}{1} & \multirow{4}{*}{ SAP1 } & $194^{\circ} \mathrm{C} 5 \mathrm{~min}$ & \multirow{8}{*}{1} & \multirow{8}{*}{$\begin{array}{c}\text { SSP1 } \\
\text { GSP1 } \\
\text { (GSP3) }\end{array}$} & 1 & $94^{\circ} \mathrm{C} 5 \min$ \\
\hline & & $30^{\circ} \mathrm{C} 3 \min$ & & & 10 & $94^{\circ} \mathrm{C} 30 \mathrm{~s}$ \\
\hline & & ramp to $65^{\circ} \mathrm{C}$ & & & \multirow{3}{*}{1} & $60^{\circ} \mathrm{C} 1 \mathrm{~min}$ \\
\hline & & at the rate of $0.25^{\circ} \mathrm{C} / \mathrm{min}$ & & & & $72^{\circ} \mathrm{C} 3 \mathrm{~min}$ \\
\hline \multirow{4}{*}{2} & \multirow{4}{*}{$\begin{array}{c}\text { GSP1 } \\
\text { (GSP3) }\end{array}$} & $65^{\circ} \mathrm{C} 5 \min$ & & & & $94^{\circ} \mathrm{C} 30 \mathrm{~s}$ \\
\hline & & $1094^{\circ} \mathrm{C} 30 \mathrm{~s}$ & & & & $35^{\circ} \mathrm{C} 3 \min$ \\
\hline & & $65^{\circ} \mathrm{C} 3 \min$ & & & & ramp to $65^{\circ} \mathrm{C}$ \\
\hline & & $172^{\circ} \mathrm{C} 5 \mathrm{~min}$ & & & at th & $\begin{array}{l}\text { e rate of } 0.25^{\circ} \mathrm{C} / \mathrm{min} \\
72^{\circ} \mathrm{C} 5 \mathrm{~min}\end{array}$ \\
\hline \multirow[b]{2}{*}{3} & \multirow[t]{2}{*}{ SAP2 } & $94^{\circ} \mathrm{C} 2 \mathrm{~min}$ & & \multirow[b]{2}{*}{ SSP2 } & 1 & $94^{\circ} \mathrm{C} 2 \mathrm{~min}$ \\
\hline & & $\begin{array}{ll}20 & 94^{\circ} \mathrm{C} 30 \mathrm{~s} \\
& 65^{\circ} \mathrm{C} 3 \mathrm{~min}\end{array}$ & 2 & & 20 & $\begin{array}{l}94^{\circ} \mathrm{C} 30 \mathrm{~s} \\
60^{\circ} \mathrm{C} 1 \mathrm{~min}\end{array}$ \\
\hline \multirow{5}{*}{4} & & $172^{\circ} \mathrm{C} 5 \mathrm{~min}$ & \multirow{5}{*}{3} & \multirow{5}{*}{$\begin{array}{c}\text { GSP2 } \\
\text { (GSP4) } \\
\text { SSP2 }\end{array}$} & 1 & $72^{\circ} \mathrm{C} 7 \min$ \\
\hline & GSP2 & $194^{\circ} \mathrm{C} 2 \mathrm{~min}$ & & & 1 & $94^{\circ} \mathrm{C} 2 \mathrm{~min}$ \\
\hline & (GSP4) & $3094^{\circ} \mathrm{C} 30 \mathrm{~s}$ & & & 30 & $94^{\circ} \mathrm{C} 30 \mathrm{~s}$ \\
\hline & SAP2 & $65^{\circ} \mathrm{C} 3 \min$ & & & & $60^{\circ} \mathrm{C} 1 \mathrm{~min}$ \\
\hline & & $172^{\circ} \mathrm{C} 5 \mathrm{~min}$ & & & 1 & $72^{\circ} \mathrm{C} 7 \mathrm{~min}$ \\
\hline
\end{tabular}

\section{RESULTS}

\section{The principle of NPA-PCR}

The principle of NPA-PCR is illustrated in Figure 1. Four primers were used in NPA-PCR. Two sequence anchoring primer (SAP), SAP1 and SAP2 (a part of SAP1), were as the nonspecific anchoring. Two gene specific primers (GSP), GSP1 and GSP2, were nested primers (Fig. 1a). Four steps were included in NPA-PCR. First, a single cycle of PCR was carried out at a low annealing temperature with only primer SAP1. At this temperature, SAP1 could prime at target or non-target positions on the DNA template (Fig. $1 b)$. Then the annealing temperature was increased to the point corresponding to the annealing temperature of GSP1. GSP1 was added at this time the amplification products were produced when SAP1 hybridized with target position by SAP1 and GSP1, meanwhile the non-target gene amplification was suppressed by the stem-loop structure of the DNA (Fig. 1c). SAP2 was added in the third step. The amplification was carried out by GSP1 and SAP2 to generate the template (Fig. 1d). The products of third step were diluted and used as template in the last step. Target DNA was exponentially amplified by the nested PCR with GSP2 and SAP2 (Fig. 1e). NPA-PCR and NASS-PCR were carried out according the protocol described above. The PCR products were separated on $1.2 \%$ agarose gel and examined (Fig. 2). The amplification results were the same for the upstream walking by NPA-PCR and NASS-PCR. Bands with the length of approximately $1500 \mathrm{bp}$ were obtained. Different results were obtained at downstream walking: a band of approximately 700bp by NPA-PCR while $600 \mathrm{bp}$ by NASS-PCR. The products were purified and sequenced.

\section{Sequence analysis}

Upstreamed 1474bp-fragments were obtained both by NPA-PCR and NASS-PCR. The sequences were the same except for the primer sequences of SAP1 and SSP1. The walking sequences had 86bp overlap with known sequence. A fragment of $701 \mathrm{bp}$ was obtained by NPA-PCR at the downstream with 70bp overlap to the known one and was consistently with design. A 2716bpfragment obtained by assembling the walking sequence and the original sequence was shown below. The sequence had $79 \%$ identity with actinomycin synthetase III $(\mathrm{acmC})$ gene cluster of Streptomyces chrysomallus, which was consistent with the antibiotic kind of $S$. setonii Z-L-22. A downstream 641-bp fragment was obtained at the end by NASS-PCR. The sequence with SSP1 at both ends showed no overlap with the known one and no similarity with any other sequences by NCBI blast analysis. It should be non-specific amplification sequence. 


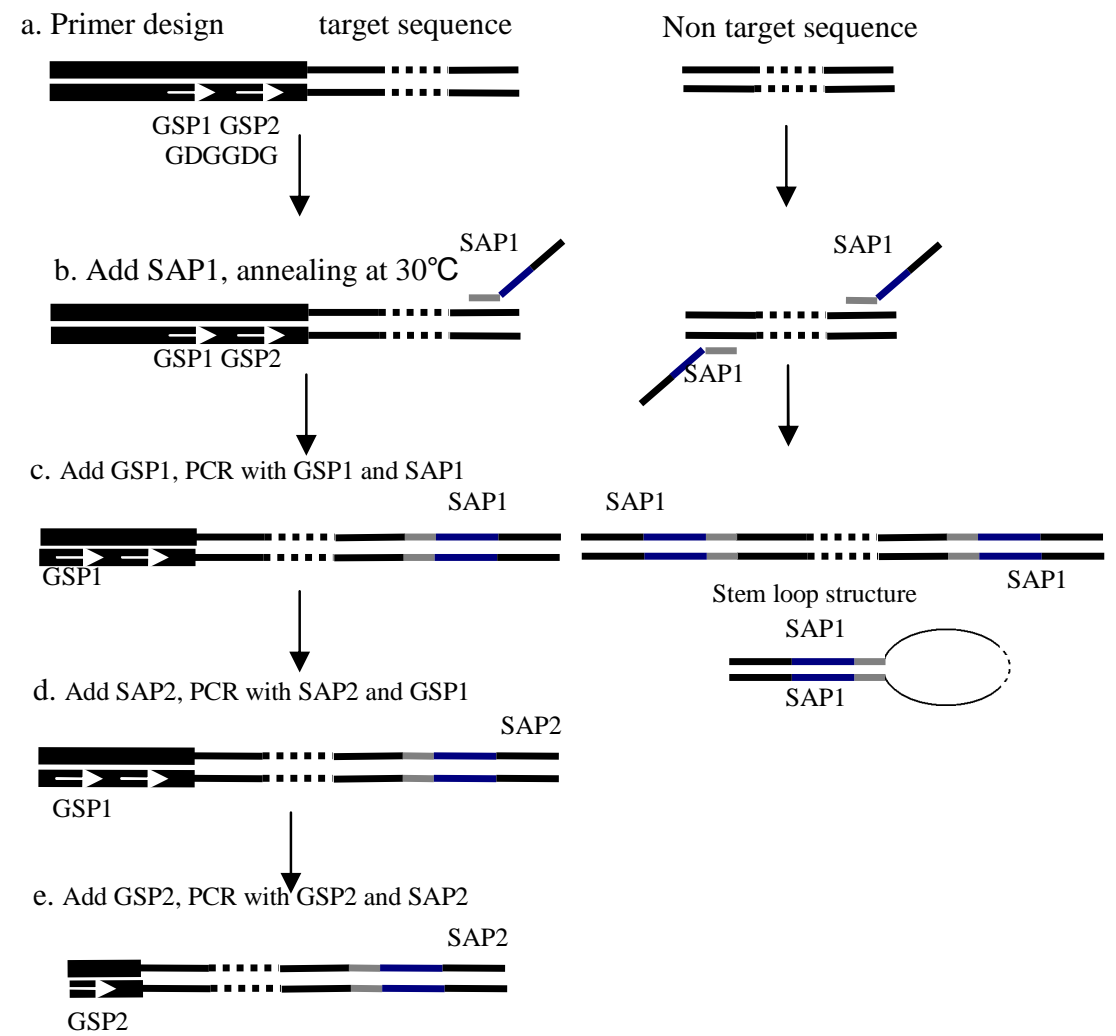

Figure 1 - Schematic outline of NPA-PCR method for chromosome walking Known and unknown sequences are depicted with thick and thin lines, respectively. DNA sequences with thin white arrows in them represent the same sequences as the primers. SAP1 is composed of grey, blue and black segments. Grey segments show the expected specific hybridized sites, black segments show the same part of SAP1 and SAP2, blue segments show the nonspecific hybridized parts particular in SAP1.

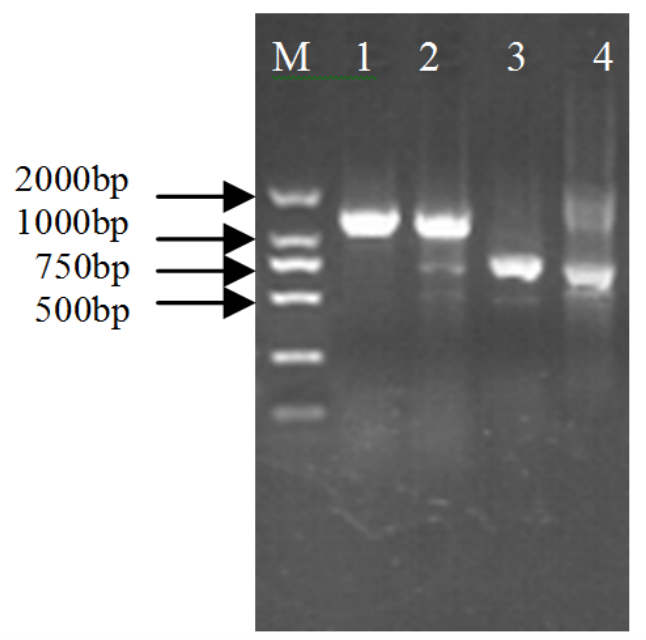

Figure 2 - Amplification results of genomic DNA of Streptomyces setonii Z-L-22 by NPA-PCR and NASS-PCR M, DNA ladder: DL2000. 1: upstream walking by NPA-PCR; 2: upstream walking by NASS-PCR; 3: downstream walking by NPA-PCR; 4: downstream walking by NPA-PCR. 
CCTGAGACCTATTACCGCCCAGAGGATCTCCGTGTCCTCTGCATAACGAACTCCTCGGCGA SAP1

CCAGAACGACCCCCACAGCCTCTTCACCAACCAGATCACCCACTGGAAAAACACCCTCGAA AACCTCCCCGACCACATCACCATCCCCACCGACCGACCCCGCCCCACCATCACCACCTACCA AGGCGACTACACCACCATCACCATCAACCCCACCCTCCACCAAAAAATCACCCACCTCGCCC ACACCACCAACACCACCGTCTTCATGGTCCTCCAAGCAGGACTCACCACCCTCCTCACCAAG CTCGGCGCCGGAAACGACATCCCCATCGGCACCCCCATCGCCGGACGCACCGACCACAACC TCGACAACCTCATCGGCTTCTTCGTCAACACCCTCGTCCTCCGCACCGACACCACCAACAAC CCCACCTTCACCCAACTCATCAACCGCATCCGCGAAACCAACCTCACCGCCTACGCCCACCA AGACGTCCCCTTCGAATACCTCGTCGAAATCCTCAACCCCACCCGCACCCTCAACCACCACC CCCTCTTCCAAATAATGCTCGCCCTCCAAAACGCACCCGAAGGAGCATTCCAACTCCCCGGC CTCACCATCGACATCGCCCCCGGACGCACCGGAACCGCCAAATTCGATCTCTTCTTCAGCCT CGTCGAGAAACGCGGACCCCACGGCGAACCCGAAGGCATCACCGGCGCCATCGAATACTCC AGCGACCTCTACGACGAAACCACCGTCCACACCCTCTTCGAACGCCTCACCCGCATCCTCGA AACCGCCACCGACCACCCCGACCAACCCCTCAGCCACATCGACGCCCTCAGCCCGCACGAG AAGCACCAGGTGCTGGACTCCTGGCTGGAGACGGCGACGGAGGTAGGGGCCGGTCTGCTGC CTGCCCGCTTCTCAGAGCAAGCGTCCGCGACACCGGACGCCGTGTCCCTGGTCGCCGGCGAC ACCGTCCTGACCTATGCCGAACTGGACCGGCGGGCCAACCGGCTGGCCCGAGTCCTGCTCGA CCGAGGTGCGGGAGCCGGCCGTGTGACCGCCATCGCGCTGCCGCGCTCGGCCGACCTGGTG GTGGCACTGCTGGCAGTGCTCAAGTCCGGCGGCGCCTACCTGCCGCTCGACCCCGACCACCC GGCGGCACGCCTTACGCACGTCCTGGAGGATGCCCGCCCCTCACTGCTGCTCACGACCACGG CGACGGACTGGATGATCCCGGAGGTGGGCGCCGCACAGCGGCTGGTCCTCGATTCCGACCC CGTACGGGAGGCATCGGACGCCGCCCTCGACAGCGATCCGGCCGACGACGGCGGGGTCGCC CCCCTCCGGCCGGAGGACGCCGCGTACGTCATCTACACCTCGGGCTCGCACCGGCCGGCCGA AGGGCGTGGTGGTCCCGCACGGCGCCTTGCTCAACTTCCTCGTGGGCATGCGGGAGAAGGCT CCGATCCGGCCGGAGGACCGGCTGCTC GSP2

GCGGTCACCACCGTCGCCTTCGACATCGCCGCCCTCGAGCTCTACCACCCCCTGCTCTCCGGC GCCGCCGTCGTCATCGCACCCAAGGAAGCCGTTCCGCAGCCCTCGGCCGTGCTGGACCTGAT CGAACGGCACAGCGTCACGGTCGTGCAGGGCACCCCGTCCCTGTGGCAACTGCTCGTGGCGC ACGACGCGGAGGCGCTTCGAGGGCTACGGATACTGGTCGGCGGCGAAGCGCTGCCGGTCTC CCTCGCCGAGGCCCTGCGCGGGCTCACCGACGACCTGGTCAACCTGTACGGCCCGACCGAG ACCACCATCTGGTCGACCGCTGCGGATCTGGCCGGCCACACCGGAGGGCCGGCACCGATCG GGCGGCCGATCGCAAACACCCGGGTCTACGTGCTGGACTCCGCTCTGCAGCCGGCCGCTCCC GGTGTGGTGGGTGAGCTGTACGTCGCGGGCGAGGGCCTGGCCCGCGGCTACGCCAACCAGC CCGGGCTCACCGCCGAACGCTTCGTCGCAGATCCGTTCCACCCCGAACCCGGGCAACGGATG 
TACCGCACCGGCGACCTCGCCCGCTGGAACACCCACGGCGAACTCGAATACCTCGGCCGTAC CGA

GSP4

CCACCAGGTCAAGATGCGGGGCTACCGCATCGAACCCGGCGAGATCGAGAAGACCCTCACC GACCACCCCGACATCGCCCAGGCCGCCGTGATCGTACGGGAGGACAGGCCGGGAGACCTCC GCCTGGTCGGGTACGTCGTGGCCGACAGCGGGGGCGGAGTCCGTGACGAGCATGTCGAGCG GGACCAGCTGAGCGAGTGGCAGGACCTCTACGACTCCGTCTACACGTCCGCCGGCGAAACT GACATCGGCGAGAACTTCGCGAGCTGGAACAGCAGTTACGACGGTCAGCCCATCCCACTGG AAGACATGCGGGAATGGCGCGACAGCACCGTCGACCGCATCAAAGCACTCCACCCACGACG CGTCCTGGAAATCGGCGTCGGCACCGGCCTCCTGCTCGCCAAAATCGCACCGGACTGCGACG AATACTGGGGAACCGACTTCTCCCCGACCGTCATCGAAGACCTACGACACCACATAGAAGC CGACCCCCACCTCACCGAGAAAGTCACCCTCCGCACCCAAGCCGCACACGAACACGGCGAC CTCAAGAAGAACCACTTCGACACCATCATCCTCAACTCCGTCGTGCAGTACTTCCCCAACGC CGGATGCATGGAGCTGCCTCTAGGAGACCCGCCATTATCCAGAGTCC

SAP1

Amplification of the assembling sequence from genome DNA

The PCR were carried out using the gene specific primers designed from the assembling sequence with genome DNA to verify the walking results.
The amplification results are shown in Figure 3. Expected fragments were $2100 \mathrm{bp}$ and $1300 \mathrm{bp}$. The amplification fragments shown in the gel were consistent with the expected. Sequencing results were same as the expected.

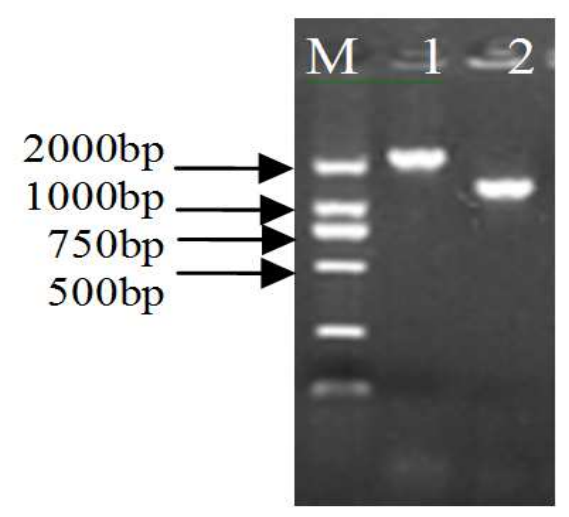

Figure 3 - Validation the walking results by amplification M, DNA ladder marker, DL2000. 1: Validation the assembling sequence at 5' by amplification; 2: Validation the assembling sequence at 5 ' by amplification. 


\section{DISCUSSION}

Anchored PCR is often used for the amplification of cDNA ends due to the same homooligonucleotide at 3'ends of mRNA (Ohara et al., 1989; Frohman et al., 1988) and generally could get good results. However, the DNA structures are not same, anchored PCR can't be used in DNA without any manipulation. Nonspecific anchoring primer, equal to adding the same structure to DNA, was used in this work, which could accomplish the amplification of unknown region.

Chromosome walking methods based on the random primer is well accepted due to simplicity and specialty (Jiang et al., 2007; Tan et al., 2005). The NPA-PCR method described in this work was designed on the basis of NASS-PCR. Random primers were redesigned and reactions were improved. NPA-PCR showed a higher specialty when employed in chromosome walking in Streptomyces setonii. The method was based upon the principle that a primer may initiate PCR at any sequences that had only partial homology to the $3^{\prime}$ end. Chromosome walking was accomplished by using two gene specific primers (GSP) which annealed to the known sequence, and a nonspecific anchoring primer (SAP1) which had sequences to be complement to a particular region that were not known near the target sequence. SAP1 hybridized to multiple sites on both the coding and complementary strands of template DNA. Targeted PCR products would only be produced when SAP1 primer annealed to a DNA strand that was contiguous with and complementary to the strand of DNA to which gene specific primer had hybridized.

SAP1, a partially degenerate primer, plays a key role in the process. It bears the effect of priming, anchoring by nonspecific hybridization and suppressing amplification of non-target sequence. The oligonucleotides 5'-NNNNNTGCAT-3' at the 3'end of SAP1 were utilized to find the TGCAT sites on the template molecules at low temperature with the help of random nucleotides NNNNN (Tan et al., 2005). Non-target molecule, amplified by single primer SAP1, contained SAP1 sequences at both the ends. The ends of the single non-target strands formed a stem-loop structure when annealing during every cycle in the second step. Stem-loop structures, stable than the primertemplate hybrid, suppressed the amplification. The length of SAP1 (44bp) facilitated the formation of PCR suppression effect.
For SAP1, as random primer, the length and kind of oligonucleotide could be varied. Different number and kinds of specific anchoring oligonucleotides at 3' ends of SAP1 would lead to different amplification results. Five specific oligonucleotides were chosen in this work and a sequence of $1474 \mathrm{bp}$ was obtained in upstream chromosome walking in $S$. setonii Z-L-22. Analyzing the sequence between SAP2 and GSP2, TGCAT sites appeared once thus creating one clear band in the gel electrophoresis. There would be more TGCAT sites of contiguous DNA far from the target sequence, which wasn't be amplified due to the length. To be addressed, two aspects increasing the specificity should be noticed in NPA-PCR in the second step. First, the concentration of GSP1 should be low while the concentration of SAP1 should be high (Wang et al., 2007). Second, GSP1 should be added at its annealing temperature.

\section{ACKNOWLEDGEMENTS}

This work was supported by doctor's foundation of Hebei province (05547005D-1)

\section{REFERENCES}

Chen, B.J., Sun, C., Wang, Y., Hu, Y. L., Lin, Z. P. (2004), Anchored PCR (A-PCR): a new method for chromosome walking. Chin Sci Bull, 49, 1772-1774

Espelund, M., Jakobsen, K. S. (1988), Cloning and direct sequencing of plant promoters using primeradapter mediated PCR on DNA coupled to a magnetic solid phase. BioTechniques, 13, 74-81

Frohman, M. A., Dush, M. K., Martin, G. R. (1988), Rapid production of full-length cDNAs from rare transcripts: Amplification using a single gene-specific oligonucleotide primer. Proc. Natl. Acad. Sci. USA, 85, 8998-9002

Jiang, Y, Pei, J. J., Song, X., Shao, W.L. (2007), Restriction Site-dependent PCR: An Efficient Technique for Fast Cloning of New Genes of Microorganisms. DNA Research, 73, 1-8

Kieser, T., Bibb, M. J., Buttner, M. J., Chater, K. F., Hopwood, D. A. (2000), Preparation and analysis of genomic and plasmid DNA. Practical Streptomyces Genetics. John Innes Foundation Norwich, England, pp. 169-171

Kilstrup, M., Kristiansen, K. N. (2000), Rapid genome walking: a simplified oligo-cassette mediated polymerase chain reaction using a single genomespecific primer. Nucleic Acids Res, 28, 1-4 
Liu, Y. G., Whittier, R. F. (1995), Thermal asymmetric interlaced PCR: automatable amplification and sequencing of insert end fragments from P1 and YAC clones for chromosome walking. Genomics, 1995, 25, 674-681

Melanie, K., Williams, R. S., Harwood, A. J. (2004), An inverse PCR technique to rapidly isolate the flanking DNA of dictyostelium insertion mutants. Molecular Biotechnology, 26, 221-224

Ochman, H., Gerber, A. S., Hartl, D. L. (1988), Genetic applications of an inverse polymerase chain reaction. Genetics, 120, 621-623

Ohara, O., Dorit, R. L., Gilbert, W. (1989), One-sided polymerase chain reaction: the amplification of cDNA. Proc. Natl. Acad. Sci. USA, 86, 5673-5677.

Peng, H. Z. (2005), Studies on the characteristic of meristem development and cloning and functional analysis of related genes in bamboo. PhD Thesis, Zhejiang University, Zhejiang, China

Tan, G.H., Gao, Y., Shi, M., Zhang, X.Y., He, S.P., Chen, Z.L., An, C.C. (2005), Site finding-PCR: a simple and efficient PCR method for chromosome walking. Nucleic Acids Res, 33, e122
Triglia, T., Peterson, M.G., Kemp, D.J. (1988), A procedure for in vitro amplification of DNA segments that lie outside the boundaries of known Sequences. Nucleic Acids Res, 16, 8186

Wang, S.M., He, J., Cui, Z.L., Li, S.P. (2007), Selfformed adaptor PCR: a Simple and Efficient Method for Chromosome Walking. Applied and Environmental Microbiology, 73, 5048-5051

Yan, Y. X., An, C. C., Li, L., Gu, J.Y., Tan, G. H., Chen, Z. L. (2003), T-linker-specific ligation PCR (T-linker PCR): an advanced PCR technique for chromosome walking or for isolation of tagged DNA ends. Nucleic Acids Res, 31, e68

Received: March 18, 2009; Revised: August 18, 2009; Accepted: April 22, 2010. 